\title{
LEVANTAMENTO FITOSSOCIOLÓGICO DA VEGETAÇÃO HERBÁCEA-SUBARBUSTIVA DAS DUNAS DA PRAIA DE MASSARANDUPIÓ, MUNICÍPIO DE ENTRE RIOS, BAHIA
}

\author{
Laene Paixão da Silva ${ }^{1}$, Ingrid Fabiana Fonseca Amorim² (D), Eduardo Bezerra de Almeida Jr. ${ }^{3}$ (D), Valdira \\ de Jesus Santos ${ }^{*}$ (iD
}

\footnotetext{
${ }^{1}$ Departamento de Educação - Campus VII, Universidade do Estado da Bahia, Rodovia Lomanto Junior, BR 407, Km 127, Senhor do Bonfim, BA, Brasil - In memoriam
}

${ }^{2}$ Programa de Pós-Graduação Biodiversidade e Biotecnologia da Amazônia Legal (BIONORTE), Universidade Federal do Maranhão, Cidade Universitária Dom Delgado, Av. dos Portugueses, 1960, São Luís, MA, Brasil.

${ }^{3}$ Departamento de Biologia, Universidade Federal do Maranhão, Cidade Universitária Dom Delgado, Av. dos Portugueses, 1966, São Luís, MA, Brasil.

${ }^{4}$ Universidade do Estado da Bahia - Laboratório de Botânica, Campus VII, Rod. Lomanto Junior BR 407 Km 127, Senhor do Bonfim, BA, Brasil.

*Autor correspondente: valjsantos@gmail.com

\section{RESUMO}

Este estudo teve como objetivo realizar uma análise fitossociológica da composição herbácea e subarbustiva, das dunas no litoral norte da Bahia, com a finalidade de conhecer e caracterizar a comunidade do estrato herbáceo-subarbustivo. A área de estudo está localizada na praia de Massarandupió (12 $\left.{ }^{\circ} 19^{\prime} 12^{\prime} \mathrm{S} ; 3^{\circ} 50^{\prime} 15^{\prime} \mathrm{W}\right)$, município de Entre Rios, Bahia. Para amostragem, foram alocadas 50 parcelas de $1 \mathrm{~m}^{2}$ ao longo da área e calculados os dados fitossociológico, o índice de diversidade Shannon $\left(\mathrm{H}^{\prime}\right)$, equabilidade de Pielou $\left(\mathrm{J}^{\prime}\right)$ e riqueza total das espécies. Foram incluídos todos os indivíduos com hábito herbáceo e subarbustivo, sendo coletados 3.804 indivíduos, pertencentes a 12 espécies, distribuídas em nove famílias. As espécies Rhynchospora riparia, Chamaecrista ramosa, Eriocaulon sp. e Comolia ovalifolia apresentaram os maiores valores de importância (VI's). Os valores de (H') e (J') quando calculados com a cobertura vegetal foram, respectivamente, $\mathrm{H}^{\prime} \mathrm{c}=1,453 \mathrm{nas} / \mathrm{m}^{2}$ e J'c $=0,585$. As dunas de Massarandupió apresentaram uma vegetação com ervas e subarbustos distribuídos em moitas. As famílias Fabaceae, Cyperaceae e Poaceae destacaram-se por apresentarem maior número de espécies, sendo predominantes em ambientes de praias, por se adaptarem facilmente em ambientes adversos assim como seu importante papel ecológico, auxiliando na fixação das dunas.

Palavras-chave: Antropização, estrutura herbácea, Restinga, Vegetação costeira do Nordeste.

PHYTOSOCIOLOGICAL SURVEY OF HERBACEOUS AND SUB-SHRUB VEGETATION OF THE MASSARANDUPIÓ BEACH DUNES, MUNICIPALITY OF ENTRE RIOS, BAHIA STATE

\footnotetext{
ABSTRACT

This study aimed to carry out a phytosociological analysis of the herbaceous and sub-shrub composition of the dunes on the northern coast of Bahia, with the purpose of knowing and characterizing the herbaceous-subshrub community. The study area is located on the beach of Massarandupió $\left(12^{\circ} 19^{\prime} 12^{\prime \prime S} ; 7^{\circ} 5015^{\prime \prime} \mathrm{W}\right)$, municipality of Entre Rios, Bahia state. For sampling 50 plots of $1 \mathrm{~m} 2$ were allocated over the area and the phytosociological data, Shannon diversity index $\left(\mathrm{H}^{\prime}\right)$, Pielou equability $\left(\mathrm{J}^{\prime}\right)$ and total species richness were calculated. The individuals with herbaceous and sub-shrub habit were included in the present study. Were collected 3,804 individuals, belonging to 12 species, distributed in nine families. The species Rhynchospora riparia, Chamaecrista ramosa, Eriocaulon sp. and Comolia ovalifolia presented the highest importance values (IV's). The values of $\left(\mathrm{H}^{\prime}\right)$ and $\left(\mathrm{J}^{\prime}\right)$ when calculated with the vegetation cover were respectively, $\mathrm{H}^{\prime} \mathrm{c}=1.453 \mathrm{nas} / \mathrm{m}^{2}$ and $\mathrm{J} ' \mathrm{c}=0.585$. Massarandupió dunes presented vegetation with herbs and sub-shrubs distributed in thickets. The families Fabaceae, Cyperaceae and Poaceae stood out for presenting a greater number of species, being predominant in beach environments, for adapting easily in harsh environments as well as their important ecological role, assisting in the fixation of dunes.

Keywords: Anthropization, herbaceous structure, Restinga, Northeast coastal vegetation.
} 


\section{INTRODUÇÃO}

As áreas litorâneas sempre despertaram grande interesse científico, uma vez que apresentam uma vegetação peculiar, compondo paisagens de restingas e dunas que englobam campos herbáceos, formações arbustivas inundáveis e não inundáveis e matas com porte médio a elevado (Lima et al., 2015). As dunas litorâneas são faixas de areia que se limitam entre os continentes e os oceanos, representando uma das áreas mais dinâmicas do planeta. As planícies costeiras brasileiras são conhecidas por serem bastante heterogêneas tanto em relação à florística quanto ao arranjo estrutural da vegetação (Suguio, 2003; Cantarelli et al., 2012).

Ao longo do litoral brasileiro ainda são poucos os estudos realizados com a vegetação herbácea, levando em consideração que a falta de conhecimento desse estrato pode comprometer o conhecimento florístico e estrutural das áreas de dunas e restingas (Schlickmann et al., 2019). Além disso, o estrato herbáceo é o componente vegetal que melhor representa as áreas de dunas, pois pode ser apontado como indicador de alterações ambientais, diante da maior fragilidade em relação aos demais estratos (Citadini-Zanette \& Baptista, 1989; Pereira et al., 2004; Santos-Filho et al., 2010).

No litoral Nordestino, dentre os estudos que tratam da composição herbácea, destacam-se os estudos da Bahia, desenvolvidos por Dias \& Menezes (2007), Nolasco et al. (2012) e Menezes et al. (2012); em Pernambuco, Almeida Jr. et al. (prelo); no Piauí, Moura et al. (2019) e no Maranhão, listam-se os estudos de Amorim et al. (2016), Araújo et al. (2016); Amorim (2017) e Santos et al. (2019).

As áreas de vegetação do litoral baiano (dunas e restingas) distribuem-se de maneira pouco uniforme devido à proximidade dos tabuleiros litorâneos da linha de praia, compreendendo formações vegetais distintas do ponto de vista fisionômico e florístico (Thomas, 2003). Apesar da sua importância ecológica, muitos aspectos da biodiversidade e conservação das dunas e restingas ainda são pouco conhecidos para o Estado. Esse fato se agrava por esse ecossistema estar situado em áreas com alta densidade humana, ocasionando uma grande pressão e acelerando o processo de degradação desses ambientes, o que pode levar à extinção de muitas espécies (Rocha et al., 2004; Barcelos et al., 2012; Silva \& Menezes, 2012).

A perda da diversidade biológica abrange diversos aspectos, entre eles, sociais, econômicos, culturais e científicos, que é acelerada devido ao aumento excessivo da população humana e pela distribuição desigual da riqueza, demostrando a necessidade de ações para o manejo das zonas litorâneas, em especial nas áreas altamente afetadas por atividades antrópicas (Menezes \& Araujo, 1999; Silva \& Menezes, 2012). Essas atividades têm levado à diminuição da vegetação, tornando esses ambientes ainda mais vulneráveis à erosão causada pelos fatores abióticos (Dewhurst, 2002; Schlickmann et al., 2019).

Deste modo, este trabalho teve como objetivo descrever a estrutura do estrato herbáceo de uma área de dunas no litoral norte da Bahia a partir dos parâmetros fitossociológicos, além de identificar as espécies dominantes na área.

\section{MATERIAL E MÉTODOS}

\section{Área de estudo}

A amostragem fitossociológica ocorreu no período de 2015 a 2016 nas dunas da praia de Massarandupió (12¹9'12”S; 3750’15”W), inserida na Área de Proteção Ambiental (APA) do Litoral Norte, no município de Entre Rios, Bahia (Figura 1). O município possui grande importância turística, por conter uma ampla variedade de ambientes naturais (restinga e dunas) (Figura 2), com uma grande riqueza florística do domínio Atlântico (Silva \& Menezes, 2012).

O clima da região é do tipo Aw, tropical quente e úmido (Alvares et al., 2013), com temperatura média anual entre $20^{\circ} \mathrm{C} \mathrm{e} 28^{\circ} \mathrm{C}$, caracterizando-se por apresentar médias térmicas elevadas, e altos índices pluviométricos distribuídos regularmente ao longo de todos os meses do ano (INMET, 2019).

\section{Coleta e análise dos dados fitossociológicos}

As coletas foram realizadas em uma área de dunas com predomínio de vegetação herbácea e subarbustiva. Para realização do estudo fitossociológico foi utilizado o método de parcelas (Mueller-Dombois \& Ellenberg, 1974; Ara ujo et al., 2016). As parcelas foram alocadas em cinco transectos de $100 \mathrm{~m}$ distribuídos ao longo da área, paralelos entre si e perpendiculares à linha da praia. Em cada transecção foram distribuídas 10 parcelas de $1 \mathrm{~m}^{2}$, posicionadas alternadamente, por meio de sorteio prévio (lado direito ou lado esquerdo), equidistantes $10 \mathrm{~m}$ entre si, totalizando 50 parcelas (Figura 3A). A cobertura da vegetação $(\mathrm{CV})$ foi estimada visualmente com auxílio de uma parcela de $1 \mathrm{~m}^{2}$ subdividida em 100 quadrículos menores de $10 \mathrm{~cm}^{2}$, o qual correspondia a $1 \%$ da parcela, sendo o critério de cobertura adotado de 0 a $100 \%$ (Figuras $3 \mathrm{~B} ; 3 \mathrm{C}$ ). 


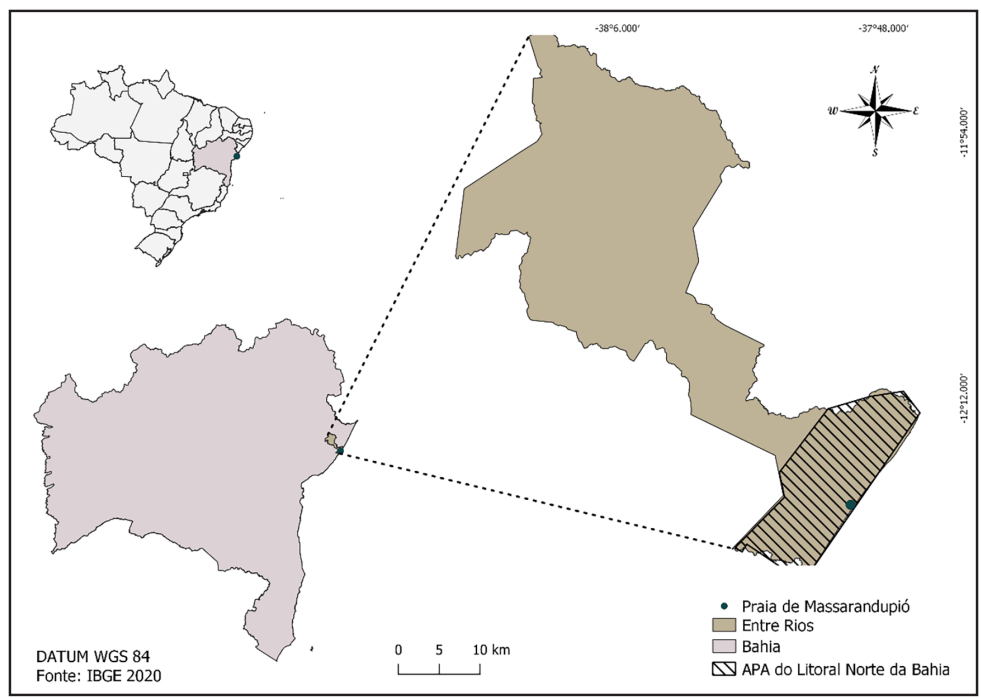

Figura 1. Localização da área de dunas em Massarandupió, município de Entre Rios, Bahia.
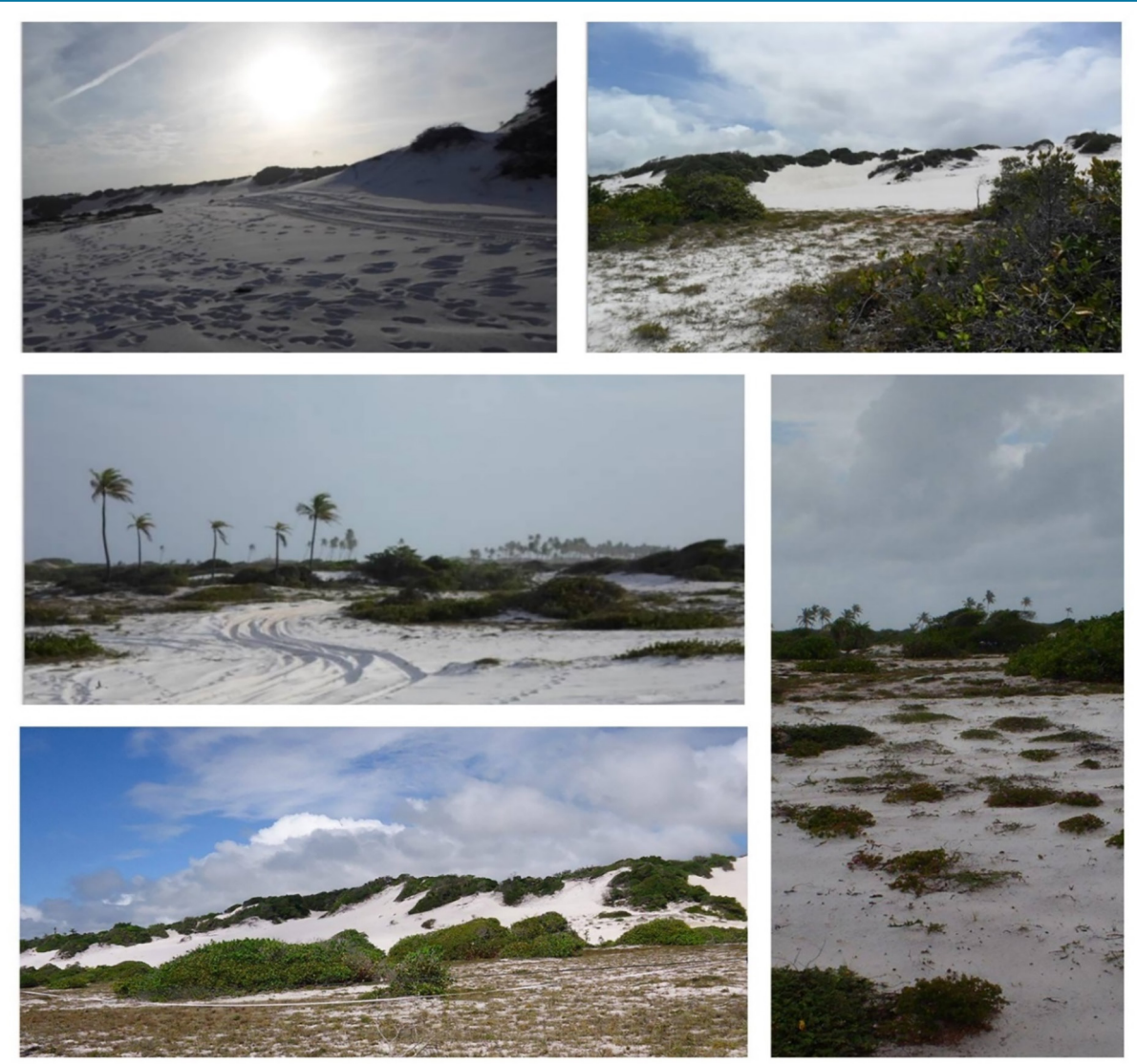

Figura 2. Região de dunas da praia de Massarandupió, Entre Rios, Bahia, onde foi realizada a amostragem fitossociológica. Fonte: V.J. Santos. 

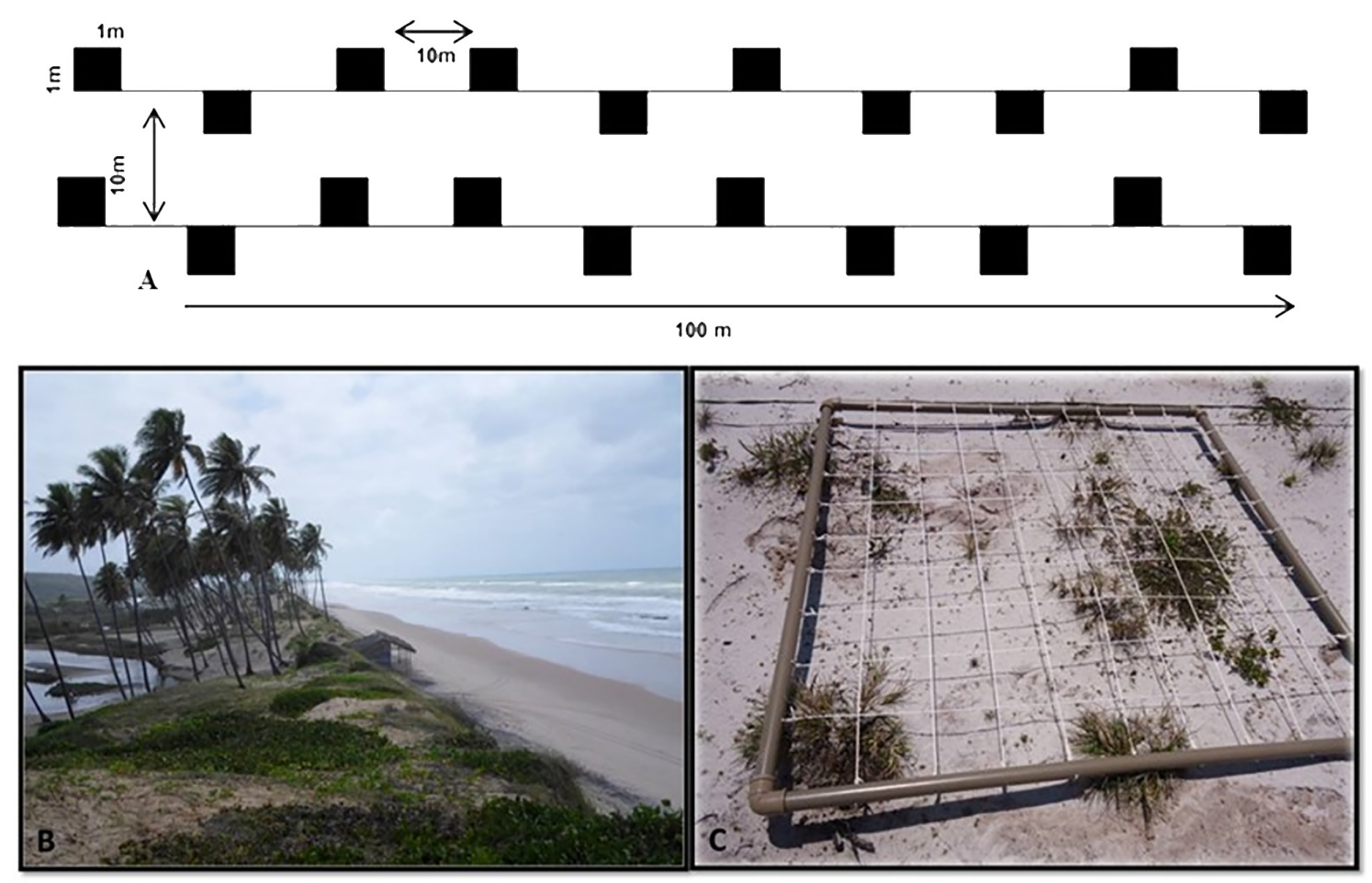

Figura 3. A) Esquema de distribuição das parcelas ao longo dos transectos; B) Imagem das formações de dunas onde foram alocadas as parcelas; C) Parcela utilizada no estudo fitossociológico.

Os indivíduos do estrato herbáceo foram classificados como ervas, subarbustos e lianas (Veloso et al., 1991; Pereira et al., 2004). Os representantes jovens de espécies arbustivas ou arbóreas não foram considerados na amostragem.

O valor de importância (VI) de cada espécie foi obtido através do somatório dos valores de frequência e cobertura relativa, excluindo-se o parâmetro densidade. Tal prática vem sendo utilizada por diferentes autores (Pereira et al., 1992; Almeida \& Araujo, 1997; Menezes \& Araujo, 1999; Cordeiro, 2005; Carvalho \& Sá, 2011) devido à dificuldade de individualização de espécimes rizomatosas e estoloníferas. A diversidade foi calculada pelo índice de Shannon (H') e a uniformidade através da equabilidade de Pielou (J'). Para ambos os índices foram utilizados os dados de cobertura e frequência das espécies como medida de abundância utilizando o pacote Microsoft $\AA$ Office Excel (2010) (Magurran, 1988).

Todos os indivíduos amostrados foram coletados segundo os critérios de Peixoto \& Maia (2013) e identificados no Laboratório de Botânica do Departamento de Educação da Universidade do Estado da Bahia - Campus VII, com auxílio de literatura especializada e comparação com exemplares pertencentes aos acervos do Herbário da Universidade do Estado da Bahia - (HUNEB), Herbário da Universidade Estadual de Feira de Santana - (HUEFS) e o Herbário Alexandre Leal Costa (ALCB) da Universidade Federal da Bahia. A organização das famílias seguiu o sistema de classificação APG IV (2016). E, ao final, todo material identificado foi incorporado ao acervo do Herbário da Universidade do Estado da Bahia HUNEB.

\section{RESULTADOS}

$\mathrm{Na}$ amostragem foram identificadas 12 espécies, nove gêneros e nove famílias, correspondendo a 3.804 indivíduos coletados na área (Tabela 1). As famílias com maior número de espécies foram Cyperaceae ( 3 espécies) e Poaceae (2 spp); as demais famílias apresentaram uma espécie, cada. As espécies que apresentaram o maior número de indivíduos foram Rhynchospora riparia (Nees) Boeckeler (2.119) e Eriocaulon sp. (719), as demais apresentaram quantidade de indivíduos inferior a 300 .

As espécies mais representativas foram Rhynchospora riparia, Chamaecrista ramosa (Vogel) H.S. Irwin \& Barneby, Eriocaulon sp. e 
Comolia ovalifolia (DC.) Triana. Rhynchospora riparia apresentou o maior valor de importância e cobertura vegetacional $(\mathrm{VI}=39,89 \%$; $\mathrm{CA}=$ $179,5 \%$ ), devido ao maior número de indivíduos. Chamaecrista ramosa obteve os segundos maiores valores de importância e cobertura relativa (VI $=37,22 \% ; \mathrm{CA}=158,9)$. Tanto $R$. riparia como C. ramosa apresentaram os mesmos valores de frequência absoluta e relativa (Tabela 1). A espécie Eriocaulon sp. apresentou o terceiro maior valor de importância (31,54\%) e Comolia ovalifolia a quarta espécie mais representativa $(\mathrm{VI}=27,73 \%)$.

O índice de Shannon, considerando a cobertura, (H'c) foi de 1,453 nats.m ${ }^{-2}$ e o de equabilidade de Pielou (J'c) 0,585. A curva de acumulação de espécies demostrou que houve uma estabilidade do número de espécies a partir da parcela 25, sugerindo que o tamanho amostral foi adequado para estimar a composição do estrato herbáceo-subarbustivo das dunas da praia de Massarandupió (Figura 4).

Tabela 1. Parâmetros fitossociológico, ordenados por valor de importância (VI), das espécies amostradas no estrato herbáceo das dunas de Massarandupió, Entre Rios, Bahia. NP - número de parcelas com ocorrência da espécie; CA cobertura absoluta; FA - frequência absoluta; CR - cobertura relativa; FR - frequência relativa; VI - valor de importância.

\begin{tabular}{l|l|c|c|c|c|c|c}
\hline \multicolumn{1}{c|}{ Espécie } & \multicolumn{1}{|c|}{ Família } & NP & CA & FA & CR & FR & VI \\
\hline $\begin{array}{l}\text { Rhynchospora riparia } \text { (Nees) Boeckeler } \\
\text { Cyperaceae }\end{array}$ & 43 & 179 & 8,6 & 23,29 & 16,6 & 39,89 \\
\hline $\begin{array}{l}\text { Chamaecrista ramosa (Vogel) H.S. Irwin \& } \\
\text { Barneby }\end{array}$ & Fabaceae & 42 & 158 & 8,6 & 20,62 & 16,6 & 37,22 \\
\hline Eriocaulon sp. & Eriocaulaceae & 41 & 121 & 8,2 & 15,71 & 15,83 & 31,54 \\
\hline Comolia ovalifolia (DC.) Triana & Melastomataceae & 37 & 103 & 7,4 & 13,45 & 14,28 & 27,73 \\
\hline Cuphea flava Spreng. & Lythraceae & 37 & 90 & 7,4 & 11,68 & 14,28 & 25,96 \\
\hline Digitaria sp. & Poaceae & 24 & 36 & 4,4 & 4,67 & 8,48 & 13,16 \\
\hline Abildgaardia baeothryon A.St.-Hil. & Cyperaceae & 19 & 26 & 3,4 & 3,43 & 6,56 & 9,99 \\
\hline Borreria verticillata (L.) G. Mey. & Rubiaceae & 7 & 17 & 1,4 & 2,27 & 2,7 & 4,97 \\
\hline Lepidaploa mucronifolia (DC.) H. Rob. & Asteraceae & 2 & 20 & 0,4 & 2,60 & 0,77 & 3,36 \\
\hline Rhynchospora barbata (Vahl) Kunth & Cyperaceae & 4 & 13 & 0,8 & 1,75 & 1,54 & 3,29 \\
\hline Indet. 3 & Indet. 3 & 3 & 2 & 0,6 & 0,32 & 1,5 & 1,47 \\
\hline $\begin{array}{l}\text { Trichanthecium cyanescens (Nees ex Trin.) } \\
\text { Zuloaga \& Morrone }\end{array}$ & Poaceae & 3 & 1 & 0,6 & 0,18 & 1,15 & 1,33 \\
\hline
\end{tabular}

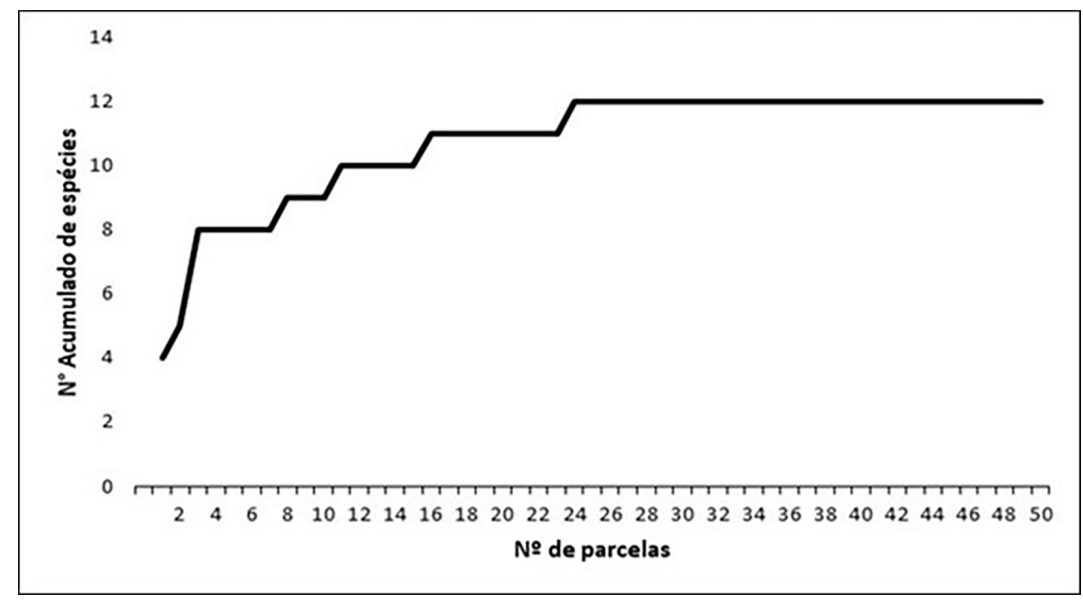

Figura 4. Curva de acumulação de espécies para amostragem nas dunas do litoral de Massarandupió, Entre Rios, Bahia. 


\section{DISCUSSÃO}

A área estudada apresenta uma vegetação herbácea e subarbustiva, distribuídas em moitas com extensão e altura variáveis, cuja vegetação herbácea ocorre em locais menos densos, intercalando as moitas. As famílias Cyperaceae e Poaceae são predominantes em ambientes de praias e estiveram entre as três famílias mais representativas; similar aos estudos já desenvolvidos em outras áreas de litoral nordestino (Dias \& Menezes, 2007; Almeida Jr. \& Zickel, 2009; Silva \& Menezes, 2012; Queiroz et al., 2012; Araújo et al., 2016; Amorim et al., 2016; Santos et al., 2019).

As espécies da família Cyperaceae apresentam facilidade em permanecer nas fisionomias abertas e com baixo nível de sombreamento, por apresentarem crescimento vegetativo e dispersão pelo vento (Cabral-Freire \& Monteiro, 1993). Cyperaceae é caracterizada por apresentar espécies pioneiras, predominantemente hemicriptófitas (CabralFreire \& Monteiro, 1993) e Poaceae, devido ao seu desenvolvimento por rizomas, por apresentar capacidade de reter nutrientes e água, permitindo sua sobrevivência nas áreas de dunas (Maciel et al., 2009; Oliveira et al., 2013).

A espécie Rhynchospora riparia é uma das mais frequentes em campos abertos, campos fechados e campos inundáveis, formando populações amplas e verdadeiras comunidades herbáceas, constituindo um mosaico de vegetação (Pereira et al., 2004; Sacramento et al., 2007). Chamaecrista ramosa é comum em ambientes costeiros e registrada em muitos levantamentos florísticos no Nordeste. As espécies de Fabaceae são importantes fixadoras de nitrogênio, proporcionando condições edáficas favoráveis ao estabelecimento de táxons mais exigentes, contribuindo, dessa forma, para uma regeneração natural da vegetação (Pereira et al., 2004; Sacramento et al., 2007; Nolasco et al., 2012; Silva \& Menezes, 2012). Borreria verticillata, Comolia ovalifolia e Cuphea flava são comuns em ambientes de dunas por estarem associadas às zonas de deflação (Silva \& Menezes, 2012), o que pode contribuir no desenvolvimento e distribuição dessas espécies ao longo do litoral.

Queiroz et al. (2012), ao realizarem um levantamento da composição florística da vegetação de restinga da APA Rio Capivara, no litoral norte da Bahia, registraram sete espécies em comum com o presente estudo, sendo elas: Borreria verticillata, Chamaecrista ramosa, Cuphea flava, Lepidaploa mucronifolia, Rhynchospora barbata, Rhynchospora riparia e Trichanthecium cyanescens. Em estudos fitossociológicos da vegetação sobre um cordãoduna no litoral norte da Bahia, Dias \& Menezes (2007) encontraram 11 espécies distribuídas em oito famílias, demostrando que o tipo de duna pode afetar diretamente a vegetação presente na região, levando em consideração as variações e movimentos das dunas e as características ecológicas das espécies vegetais que conseguem se fixar e se desenvolver.

Considerando outros estudos desenvolvidos no litoral da Bahia sobre o estrato herbáceo (Dias \& Menezes, 2007; Nolasco et al., 2012; Menezes et al., 2012), a área das dunas de Massarandupió apresentou menor riqueza de espécies. No entanto, vale ressaltar a falta de padronização metodológica entre os estudos fitossociológicos que vem sendo realizados para o estrato herbáceo, pode refletir na quantidade de espécies amostradas e consequentemente na comparação entre os dados obtidos.

Em relação aos estimadores de diversidade e equabilidade, poucos estudos apresentam esses dados em relação ao estrato herbáceo de dunas e restingas, devido à dificuldade de individualização dos espécimes que possuem crescimento clonal, comum nesses ambientes (Pereira et al., 2004), o que inviabiliza a obtenção dos valores de abundância. Apesar disso, Pereira et al. (2004) usaram o parâmetro de cobertura para avaliar a diversidade e a equabilidade, e foi observado que essa medida é a que melhor representa o estrato herbáceo (Araújo et al., 2016).

Diante disso, os valores de diversidade Shannon $\left(\mathrm{H}_{\mathrm{c}}\right.$ ) e Equabilidade ( $\left.\mathrm{J}_{\mathrm{c}}\right)$, a partir da análise da cobertura vegetal, tem sido observados nos estudos das dunas do Maranhão. Com isso, Araujo et al. (2016) estudando o estrato herbáceo das dunas da praia de São Marcos obtiveram valores de $\mathrm{H}_{\mathrm{c}}{ }_{\mathrm{c}}=1,69$ nats $/ \mathrm{m}^{2}$ e J' ${ }_{\mathrm{c}}=0,47$; Amorim et al. (2016) registraram para as dunas da Praia de Araçagi os valores de $\mathrm{H}^{\prime}=2,47$ nats $/ \mathrm{m}^{2}$ e J' ${ }_{\mathrm{c}}=0,64$; e Santos et al. (2019) ao analisarem a vegetação halófilo-psamófilo em uma área de dunas da praia São Marcos, destacaram os valores de $\mathrm{H}_{\mathrm{c}}=1,58$ nats. $\mathrm{m}^{2}, \mathrm{~J}^{\prime}{ }_{\mathrm{c}}=0,42$. Esses dados mostram que os valores obtidos no presente estudo $\left(\mathrm{H}_{\mathrm{c}}{ }_{\mathrm{c}}=1,453\right.$ nats $/ \mathrm{m}^{2} \mathrm{e} \mathrm{J}{ }_{\mathrm{c}}=$ $0,585)$ estão dentro da média registrada em relação à diversidade e equabilidade do componente herbáceo nas dunas, devido a importância do crescimento e manutenção desse estrato no litoral do Nordeste. Cabe destacar que a ausência dos valores referentes aos estimadores H' e J', de outras áreas de dunas citadas anteriormente, impossibilitaram associações entre áreas do litoral da Bahia. 
Em relação a estabilidade na curva de acumulação de espécies, nota-se que o tamanho da amostra foi considerado suficiente para caracterizar o estrato herbáceo-arbustivo nestas dunas, assim como observado em outros estudos desenvolvidos em áreas de dunas e restingas (Amorim et al., 2016; Araujo et al., 2016; Melo Júnior et al., 2017; Santos et al., 2019).

Diante disso, pode-se concluir que a composição herbácea das dunas de Massarandupió apresentou riqueza inferior em relação aos estudos realizados no litoral da Bahia, mas com índice de diversidade na média quando comparados com os trabalhos realizados nas restingas do Maranhão considerando valores de diversidade associados a cobertura. As espécies Rhynchospora riparia, Chamaecrista ramosa, Eriocaulon sp. e Comolia ovalifolia, destacaram-se em relação aos valores de cobertura e frequência. Os dados obtidos podem contribuir com ações de proteção ou conservação da vegetação de dunas da costa da Bahia diante das particularidades observadas nesse ambiente.

\section{AGRADECIMENTOS}

Agradecemos à Universidade do Estado da Bahia - UNEB - Departamento de Educação DEDC - Campus VII - pelo apoio institucional para o desenvolvimento deste estudo e pela concessão de bolsa para o primeiro autor.

\section{REFERÊNCIAS}

ALMEIDA, A.L., ARAUJO, D.S.D. 1997. Comunidades vegetais do cordão arenoso externo da reserva Ecológica estadual de Jacarepiá, Saquarema, RJ. Oecologia Brasiliensis 3(1): 47-63.

ALMEIDA JR., E.B., ZICKEL, C.S. 2009. Fisionomia psamófila-reptante: riqueza e composição de espécies na praia da Pipa, Rio Grande do Norte, Brasil. Pesquisas, Botânica 60(1): 289-299.

ALMEIDA JR., E.B., COSTA, L.B.S., OLIVO, M.A., ARAÚJO, E.L., ZICKEL, C.S. (prelo). Estrutura do componente herbáceo no campo não inundável de uma restinga em Pernambuco (Brasil). Revista Nordestina de Biologia.

ALVARES, C.A., STAPE, J.L., SENTELHAS, P.C., MORAES, G., LEONARDO, J., SPAROVEK, G. 2013. KOPPEN. W. Climate classification map for Brazil. Meteorol, 22: 711-718.

AMORIM, I.F.F. 2017. Herbáceas em áreas de dunas da Ilha do Maranhão: diversidade, riqueza e conservação. Dissertação (Mestrado), $67 \mathrm{f}$. Universidade Federal do Maranhão.

AMORIM, I.F.F., SANTOS-FILHO, F., ALMEIDA JR., E.B. 2016. Fitossociologia do estrato herbáceo de uma área de dunas em Araçagi, Maranhão. In: Biodiversidade do meio norte do Brasil: conhecimentos ecológicos e aplicações. ALMEIDA Jr., SANTOS-FILHO, F. (Organizadores), Curitiba: CRV.

APG IV. 2016. An update of the Angiosperm Phylogeny Group classification for the orders and families of flowering plants: APG IV. Botanical Journal of the Linnean Society, p. 1-20.

ARAUJO, A.C.M., SILVA, A.N.F., ALMEIDA JR, E.B. 2016. Caracterização estrutural e status de conservação do estrato herbáceo de dunas da Praia de São Marcos, Maranhão, Brasil. Acta Amazonica 46(3): 247-258.

BARCELOS, M.E.F., RIGUETE J.R., SILVA L.T.P., FERREIRA JR, P.D. 2012. Uma visão panorâmica sobre os solos das restingas e seu papel na definição de comunidades vegetais nas planícies costeiras do sudeste do Brasil. Natureza online 10(2): 71-76.

CABRAL-FREIRE, M.C., MONTEIRO, R. 1993. Florísticas das praias da Ilha de São Luiz, Estado do Maranhão (Brasil): Diversidade de espécies e suas ocorrências no litoral brasileiro. Acta amazônica. 23 (2-3): 125-140.

CANTARELli, J.R.R., ALMEIDA JR., E.B., SANTOS-FILHO, F.S., ZICKEL, C.S. 2012. Tipos fisionômicos e flora vascular da restinga da APA de Guadalupe, Pernambuco, Brasil. Insula Revista de Botânica, (41), 95-117.

CARVALHO, D.A., SÁ, C.F.C. 2011. Estrutura do estrato herbáceo de uma restinga arbustiva aberta na APA de Massambaba, Rio de Janeiro, Brasil. Rodriguésia, 62(2): 367-378.

CITADINI-ZANETTE, V., BAPTISTA, L.R.M. 1989. Vegetação herbácea terrícola de uma comunidade florestal em Limoeiro, Município de Torres, Rio Grande do Sul. Boletim do Instituto de Biociências 45: 1-87.

CORDEIRO, S.Z. 2005. Composição e distribuição da vegetação herbácea em três áreas com fisionomias distintas na Praia do Peró, Cabo Frio, RJ, Brasil. Acta Botanica Brasilica, 19(4): 679- 693.

DEWHURST, D. 2002. Coastal Dunes: Dune 
protection and improvement manual for the Texas Gulf Coast. Texas General Land Office, Austin.

DIAS, F.J.K., MENEZES, C.M. 2007. Fitossociologia da vegetação sobre um cordão-duna no Litoral Norte da Bahia, Mata de São João, Brasil. Revista Brasileira de Biociências 5(2): 1171-1173.

INMET. Instituto Nacional de Meteorologia. 2019. Disponível em: http://www.inmet.gov.br. Acesso em: 05/06/2019.

LIMA, P.B., LIMA, L.F., SANTOS, B.A., TABARELLI, M., ZICKEL, C.S. 2015. Altered herb assemblages in fragments of the Brazilian Atlantic forest. Biological Conservation, 191: 588- 595.

MACIEL, J.R., OLIVEIRA, R.C., ALVES, M. 2009. Paspalum L. (Poaceae: Panicoideae: Paniceae) no estado de Pernambuco, Brasil. Acta Botanica Brasilica, 23: 1145-1161.

MAGURRAN, A.E. 1988. Ecological diversity and its measurement. New Jersey, Princeton University Press.

MELO JÚNIOR, J.C.F., FERRARI, A., GERN, A.F., DANIEL, AL., MAIA, A.C., HARTELT, B.T., POSSAMAI, B.T., VANZUITA, C.A., DUMKE, D., SILVA, D., TUSSOLINI, E.G.R., HAFEMANN, F., SILVA, G.R., OLIVEIRA, G.B., JANTSCH, G., BATISTA, J.M., FERREIRA, J., CRUZZETA, K.M., TEIXEIRA, L.R.R.V., BURMESTER, L.P., SANTOS, M.C., SABINO, P.C., VALDEZ, R.E., JASPER, R.B., PINTO, R.F., OLIVEIRA, S., SEIFERT-NETO, V.R. 2017. Fitossociologia comparada da comunidade herbáceo-subarbustiva de restinga em duas geoformações de dunas na planície costeira de Santa Catarina. Acta Biológica Catarinense, 4(2): 5-15.

MENEZES, C.M., ESPINHEIRA, M.J.C.L., DIAS, F.J.K., SILVA, V.Í.S. 2012. Composição florística e fitossociologia de trechos da vegetação praial dos litorais norte e sul do Estado da Bahia. Revista Biociências, 18(1): 35-41.

MENEZES, L.T.F., ARAÚJO, D.S.D. 1999. Estrutura de duas formações vegetais no cordão externo da Restinga da Marambaia, RJ. Acta Botanica Brasilica, 13(2): 223-235.

MOURA, B.M.R., CRUZ, A.V.C., ARAÚJO, J.S., SANTOS-FILHO, F.S. 2019. Uma comunidade pioneira em dunas: a antropização modifica a composição florística? Revista Brasileira de Geografia Física, 12(7): 2645-2659.
MUELLER-DOMBOIS, D., ELLENBERG, H. 1974. Aims and methods of vegetation ecology. New York: John Wiley e Sons.

NOLASCO, A.P., SILVA, V.I.S., MENEZES, C.M. 2012. Aspectos florísticos e fitossociológicos da vegetação de entre-moitas em um trecho de uma restinga de Praia do Forte, município de Mata de São João, litoral norte do estado da Bahia. Revista Biociências, 18(1): 42-48.

OLIVEIRA, R.C., SANTANA, S.H., SILVA, A.S., MACIEL, J.R., VALLS, J.F.M. 2013. Paspalum (Poaceae) no Rio Grande do Norte, Brasil. Rodriguésia, 64: 847-862.

PEIXOTO, A.L., MAIA, L.C. 2013. Manual de Procedimentos para herbários. INCT-Herbário virtual para a Flora e os Fungos. Editora Universitária UFPE, Recife.

PEREIRA, M.C.A., CORDEIRO, S.Z., ARAÚJO, D.S.D. 2004. Estrutura do estrato herbáceo na formação aberta de Clusia do Parque Nacional da Restinga de Jurubatiba, RJ, Brasil. Acta Botanica Brasilica, 18(3): 677-687.

PEREIRA, O.J., THOMAZ, L.D., ARAÚJO, D.S.D. 1992. Fitossociologia da vegetação de antedunas da restinga de Setiba/Guarapari e em Interlagos/Vila Velha, ES. Boletim do Museu de Biologia Mello Leitão, 1: 65-75.

QUEIROZ, E.P., CARDOSO, D.B.O.S., FERREIRA, M.H.S. 2012. Composição florística da vegetação de restinga da APA Rio Capivara, Litoral Norte da Bahia, Brasil. Sitientibus série Ciências Biológicas, 12(1): 119-141.

ROCHA, C.F.D., VAN SLUYS, M., VRCIBRADIC, D., HATANO, F.H., GALDINO, C.A., CUNHABARROS, M., KIEFER, M.C. 2004. A comunidade de répteis na restinga de Jurubatiba. Pp. 179-198 In: Pesquisas Ecológicas de Longa Duração na Restinga de Jurubatiba: Ecologia, História Natural e Conservação. ROCHA, C.F.D., ESTEVES, F.A., SCARANO, F.R. (Organizadores), São Carlos: Rima.

SACRAMENTO, A.C.S., ZICKEL, C.S., ALMEIDA JR., E.B. 2007. Aspectos florísticos da vegetação de restinga no litoral de Pernambuco. Revista Árvore, 31(6): 1121-1130.

SANTOS, C.R., AMORIM, I.F.F., ALMEIDA JR., E.B. 2019. Caracterização fitossociológica do componente Halófilo-psamófilo em uma área de dunas, Maranhão, Brasil. Boletim do Laboratório de Hidrobiologia, 29(1):1-8. 
SANTOS-FILHO, F.S., ALMEIDA JR., E.B., SOARES, C.J.R.S., ZICKEL, C.S. 2010. Fisionomias das restingas do delta do Parnaíba, Nordeste, Brasil. Revista Brasileira de Geografia Física, 3(3): 218-227.

SCHLICKMANN, M.B., FERREIRA, M.E.A., VARELA, E.P., PEREIRA, J.L., DUARTE, E., LUZ, A.P.C.D., DREYER, J.B.B., SILVA, M.T.S., PINTO, F.M. 2019. Fitossociologia de um fragmento de restinga herbáceo-subarbustiva no sul do Estado de Santa Catarina, Brasil. Hoehnea, 46(2): e292018.

SILVA, V.I.S., MENEZES, C.M. 2012. Contribuição para o conhecimento da vegetação de restinga de Massarandupió, Município de Entre Rios, BA, Brasil. Revista da Gestão Costeira Integrada, 12(2):
239-251.

SUGUIO, K. 2003. Tópicos de geociências para o desenvolvimento sustentável: as regiões litorâneas. Revista do Instituto de Geociências - USP2: 1-40.

THOMAS, W.W. 2003. Natural vegetation types in southern Bahia. In: Corredor de Biodiversidade da Mata Atlântica do Sul da Bahia. p. 1-4. Prado, P.I., LANDAU. E. C., MOURA, R.T., PINTO, L.P.S., FONSECA, G.A.B., ALGER, K. (Organizadores). IESB/CI/CABS/UFMG/ UNICAMP.

VELOSO, H.P., RANGEL-FILHO, A.L.R., LIMA, J.C.A. 1991.Classificação da vegetação brasileira adaptada a um sistema universal. Rio de Janeiro, IBGE, Departamento de Recursos Naturais e Estudos Ambientais. 